\title{
MARKERS OF EXTRACELLULAR MATRIX AND EPITHELIAL CELL MATURATION AFTER FETAL TRACHEAL OCCLUSION IN NEWBORN RABBITS WITH LUNG HYPOPLASIA INDUCED BY DIAPHRAGMATIC HERNIA
}

\author{
A. Vuckovic ${ }^{1}$, S. Herber-Jonat ${ }^{2}$, I. Rühl ${ }^{3}$, J. Jani ${ }^{4}$, A.W. Flemmer ${ }^{2}$ \\ ${ }^{1}$ Laboratory of Physiology and Physiopathology, Université Libre de Bruxelle, Brussels, Belgium, ${ }^{2}$ Div. of \\ Neonatology, University Children's Hospital, ${ }^{3}$ Div. of Perinatology, LMU Munich, Munich, Germany, \\ ${ }^{4}$ Obstetrics and Gynecology, University Hospital Brugmann, Brussels, Belgium
}

Aim: To assess the effects of tracheal occlusion (TO) on pulmonary myofibroblast and matrix markers, TGF- $\beta$ superfamily, and epithelial maturation in a newborn model of diaphragmatic hernia (CDH).

Methods: On day 23/31, rabbit fetuses were randomized to CDH or SHAM thoracotomy. On day 28/31, the same fetuses randomly underwent TO or SHAM-TO. After ventilation at term, lungs were harvested for RTq-PCR. Non-operated littermates served as controls. Gene expressions in left lungs were expressed as fold induction relative to controls.

Results: TO increased significantly LBWR in $\mathrm{CDH}$ and SHAM-CDH animals. Data as mean \pm SD. Difference with CDH-SHAM TO $\left(^{*}\right.$ ) or SHAM CDH-SHAM TO $\left(^{+}\right)$for $\mathrm{p}<0.05$ (Kruskal-Wallis or one-way ANOVA).

\begin{tabular}{|l|l|l|l|l|}
\hline & CDH-TO & CDH-SHAM TO & SHAM CDH-TO & $\begin{array}{l}\text { SHAM CDH- } \\
\text { SHAM TO }\end{array}$ \\
\hline ELN & $3.59 \pm 2.74^{*},+$ & $0.63 \pm 0.23$ & $4.63 \pm 3.44^{*},+$ & $0.75 \pm 0.25$ \\
\hline COL1A1 & $2.32 \pm 0.65^{*},+$ & $1.29 \pm 0.69$ & $2.25 \pm 0.69^{*},+$ & $1.15 \pm 0.24$ \\
\hline DBN1 & $1.42 \pm 0.53^{*}$ & $0.67 \pm 0.22$ & $1.57 \pm 0.66^{*}$ & $0.95 \pm 0.21$ \\
\hline$\alpha$-SMA & $2.57 \pm 0.76^{*}$ & $0.87 \pm 0.37$ & $3.29 \pm 1.57^{*}$ & $0.98 \pm 0.23$ \\
\hline TGF-31 & $1.43 \pm 0.36$ & $0.91 \pm 0.33$ & $1.70 \pm 0.43^{*},+$ & $1.06 \pm 0.30$ \\
\hline TGF-32 & $1.36 \pm 0.41^{*},+$ & $0.72 \pm 0.21$ & $1.44 \pm 0.48^{*},+$ & $0.96 \pm 0.14$ \\
\hline Cav-1 & $1.68 \pm 0.63^{*}$ & $0.89 \pm 0.47$ & $2.28 \pm 0.88^{*},+$ & $1.07 \pm 0.33$ \\
\hline SP-A & $0.92 \pm 0.79$ & $1.76 \pm 0.87$ & $1.02 \pm 0.71$ & $1.24 \pm 0.50$ \\
\hline SP-B & $1.0 \pm 0.63$ & $1.47 \pm 0.45$ & $0.93 \pm 0.52$ & $1.17 \pm 0.33$ \\
\hline \hline
\end{tabular}

[Partial RTq-PCR results]

Conclusion: TO enhanced transcription of markers of myofibroblast and alveolar type II cell differentiation, together with an overexpression of TGF- $\beta$ isoforms. These data suggest that TO might induce matrix formation and tends to inhibit surfactant synthesis under the action of TGF- $\beta$. 